\title{
Wireless Space Plug-and-Play Architecture (SPA-Z)
}

\author{
Richard Alena \\ NASA Ames Research Center \\ 650-604-0262 \\ Richard.l.alena@nasa.gov
}

\author{
John Ossenfort \\ SGT Inc. \\ NASA Ames Research Center \\ John.Ossenfort@nasa.gov
}

\author{
Thom Stone \\ CSC Inc. \\ NASA Ames Research Center \\ Thom.Stone@nasa.gov
}

\author{
Jarren Baldwin \\ USRA \\ NASA Ames Research Center \\ Jarren.Baldwin@gmail.com
}

\begin{abstract}
Space Plug-and-Play Architecture (SPA), defined by the Air Force Research Laboratory, is a new standard for spacecraft component interconnections (AIAA-S-133-x-2013) providing new capability for managing intelligent components. Wireless Sensor Networks (WSN) based on the IEEE 802.15.4 Personal Area Network standard are finding increasing use in the home automation and emerging smart energy markets. The network protocol and application layers can be based on the ZigBee standard as defined by the ZigBee Alliance, providing a framework for component-based software that supports solutions from multiple vendors. SPA and ZigBee create selfconfiguring ad-hoc networks, but differ in their approach. SPA focuses on self-configuring components using wired interconnects while ZigBee forms self-configuring wireless networks. The optimal combination of SPA with ZigBee technology can bring the advantages of both methods to nextgeneration spacecraft by using self-configuring wireless networks for data and intelligent components with universal SPA-compliant interfaces. Mesh-enabled WSNs provide inherent fault tolerance and SPA provides dynamic fault management leading to low-power, low-cost ancillary sensing solutions for spacecraft.
\end{abstract}

Self-configuring architectures are the key for supporting a large number of sensors in dynamic configurations, allowing intelligent response for fault tolerant networks. Plug-and-Play for sensor networks could be defined as the capability for application software to query any sensor module connected to the ad-hoc dynamic network using module resident information defining the sensors characteristics. The embedding of sensor information into each Wireless Sensor Module (WSM) allows identifying each sensor unambiguously and accurately in terms of function and status, without the use of any configuration database. The IEEE 1451 Smart Transducer Interface Standard defines Transducer Electronic Datasheets (TEDS) containing key information regarding sensor characteristics such as name, description, serial number and calibration information. SPA defines an extensible format called xTEDS using XML embedded meta-information for sensor management enabling software to identify the sensor and interpret the sensor data stream without reference to any external information. The application software is able to read the status of each sensor module, responding in real-time to changes of WSN configuration and provide the appropriate response for maintaining overall sensor system function, even when sensor modules fail or the network is reconfigured. Temporal integrity of sensor data delivery is ensured by the use of a global network clock and embedding timestamps into each measurement result accurate to one millisecond.

SPA provides high-level mechanisms for self-configuration and integration with other spacecraft components and can significantly improve interoperability. The architecture and technical feasibility for creating wireless fault-tolerant sensor networks is presented through integration of SPA, IEEE 1451 and ZigBee into the proposed SPA-Z architecture. SPA provides the broad framework, the IEEE 1451 standards provide templates for TEDS and sensor management and ZigBee provides effective wireless network management. The approach is to tailor these multiple standards into a viable architecture. The result conforms to multiple standards, enables deterministic response and provides a capable publish/subscribe interface to application software. Our proposed software architecture for intelligent sensor management using the SPA standard will be discussed in the context of the specific tradeoffs required for effective use. Two examples are presented, the first highlights SPA- $Z$ advantages for reconfigurable payloads and the second describes the development of a SPA compliant WSN.

\section{TABLE OF CONTENTS}

1. INTRODUCTION ................................................2

2. IEEE 802.15.4 AND ZIGBEE OVERVIEW .........2

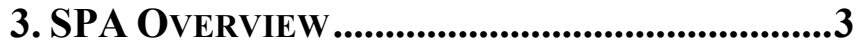

4. SPA MESSAGING .............................................4

SPA Topology Discovery ................................ 4

Component Registration ................................ 4

Subscription Processing..................................... 5

5. SPA-Z DEFINITION.........................................5

ZigBee to CAS Addressing ............................ 6

Generating xTEDS ...................................... 6

Data distribution system (DDS) ...................... 7

ZigBee Service Interface .................................. 7

6. SPA-Z FOR SPACECRAFT ...................................7

7. DEVELOPMENTAL AND FLIGHT

INSTRUMENTATION SYSTEM ................................99

Proposed DFI architecture.............................. 9

Network Discovery Implementation .............. 10

xTEDS Aggregation ..................................... 10

Middleware Implementation ......................... 10

Redundancy and Fault Tolerance. .................. 10

8. Conclusions ...........................................10

ACKNOWLEDGEMENTS....................................11

REFERENCES...........................................16

BIOGRAPHY .......................................................16 


\section{INTRODUCTION}

The primary objective of SPA-Z development is to craft a standard reference architecture that will allow rapid development of spacecraft avionics where components can be added or changed without major physical or software redesign. This architecture will include the recognized advantages of wireless systems: mass reduction, ease of installation, instrumentation of areas that are difficult to wire, electrical isolation of subsystems and high-level protocols for redundant operations and automatic data retransmission. The architecture will employ the new generation of smart sensors, actuators and other components that declare their relevant attributes when they dynamically join the wireless network. What we propose is using the best of existing standards by modifying and extending commercial hardware and software.

The Discovery and Systems Health Group at NASA Ames Research Center has been developing Wireless Sensor Network (WSN) technology for use aboard spacecraft for monitoring aerospace vehicles using funding from the NASA Engineering and Safety Center and Exploration Technology Development and Demonstration Program. This technology can be readily applied to small reconfigurable spacecraft and to Developmental and Flight Instrumentation (DFI) enabling advanced Integrated System Health (ISHM) monitoring capabilities. Mesh-enabled WSNs provide inherent fault tolerance and the Space Plug-and-Play Avionics (SPA) standards provide dynamic configuration and fault management leading to low-power, low-cost robust sensing solutions for spacecraft. The Wireless Connections in Space Project funded development of a prototype ZigBee-based sensor network for spacecraft, upgrading components to the latest Personal Area Network (PAN) radio technology, incorporating firmware enhancements such as module identity and timestamps, supporting new types of sensors and identifying shortcomings of the current implementation approaches. A miniature Wireless Sensor Module (WSM) was developed using System on a Chip (SoC) technology, resulting in an exceptionally small form-factor prototype for future flight tests. An earlier paper characterized the Radio Frequency (RF) properties of this wireless prototype system, which combined ZigBee-based frameworks with IEEE 1451 Smart Transducer Interface Standards. ${ }^{1}$

We propose to integrate different computer standards creating a new wireless sensor network technology that offers self-configuration supporting a significant scale-up of the number and type of sensors supported as well as simplifying the software required to manage and use such a network. The combination of the Air Force Research Lab (AFRL) Space Plug-and-Play Avionics (SPA) standards for next-generation spacecraft together with the IEEE 1451 Smart Transducer Interface standards and the use of the Consultative Committee for Space Data Standards (CCSDS) Spacercraft Onboard Information Services (SOIS) framework can lead to intelligent adaptable sensor networks applicable to a wide range of applications. The key innovation is the use of wireless interconnections for implementing the SPA subnet, in this case based on the IEEE 802.15.4 Personal Area Network (PAN) standard and the ZigBee Alliance wireless network framework. Currently SPA is supported on wired I2C, CAN, serial and Ethernet interfaces. This paper attempts to define the architecture and implementation approach using the appropriate elements of these complementary standards.

The paper describes wireless sensor networks applied to aerospace vehicles for two distinct applications: reconfigurable payloads and flight test instrumentation. Implementing reconfigurable payloads for spacecraft built from standard components using SPA-enabled wireless networks for payload data communication can result in rapid customization for a given mission requiring little additional software development and system integration. This application requires the development of wireless modules able to control subsystems through the wireless network. This is precisely the vision for SPA, which can be greatly improved through the use of wireless networks, by eliminating wired interconnects for data and providing flexibility for mounting within spacecraft. The Payload modules have fully SPA-compliant interfaces with metadata based functional descriptions enabling selfconfiguration. The other application Developmental and Flight Instrumentation (DFI) adds sensors for specific flight validation tests that can be left in place for ISHM and lifecycle cost optimization. The specific system and software architecture for the DFI application is presented based on our prototype implementations.

This paper is intended to further define SPA-Z implementation, which was conceptually outlined in a prior paper that mapped specific ZigBee functions into IEEE 1451 and SPA Standards. ${ }^{2}$ The current paper develops two different SPA-compliant architectures one supporting a reconfigurable satellite payload and the other a wireless DFI system. The payload subsystem is viewed as a collection of SPA-compliant components and the DFI system uses the Applique Sensor Interface Module (ASIM) approach, which hosts SPA functions only at its interface to the spacecraft. We define the functions hosted in specific components of these example SPA-Z systems and consider both hardware and software for the proposed architecture. Sample xTEDS meta-tags describing a sensor module and core SPA messages compliant with SOIS are provided to help understand the technical approach. The objective of this work is to provide a good working definition of SPA-Z while providing guidance for implementing an early prototype.

\section{IEEE 802.15.4 AND ZIGBEE OVERVIEW}

ZigBee is a network protocol and application support layer working over the IEEE 802.14.5 PAN standard for the Physical (PHY) and Media Access Control (MAC) layers. The ZigBee Alliance defines the software Framework and specific Profiles for standard functions. There are four main types of ZigBee nodes, the Coordinator that forms and 
manages the PAN, the Router which directs data traffic through the mesh network, the Gateway spanning ZigBee to other networks and the Endpoint, which hosts sensors and provides data to the wireless network. While most devices are on continuously, the endpoints can function with a very low duty cycle conserving power. The PAN can support raw data rates of up to $250 \mathrm{Kbps}^{3}$ The ZigBee protocol is supported by numerous software frameworks, which implement the details of the mesh routing and dynamic addressing protocol but allow a developer to significantly customize the network and node behavior for a specific application. ZigBee supports profiles, such as Smart Energy or Home Automation, which help standardize node definitions and network behavior. A ZigBee profile could be setup for Aerospace sensing applications, which could potentially be SPA-compliant.

ZigBee operates in the $900 \mathrm{MHz}$ and $2.4 \mathrm{GHz}$ Instrumentation, Scientific and Measurement (ISM) bands, which use unlicensed low-power transmitters supporting many concurrent users. The 802.14.5 standard specifies the use of frequency hopping spread spectrum (FHSS) modulation to limit interference, defining 16 channels for use by ZigBee within the full ISM band. Certain applications, such as Smart Meters, shift the frequency to licensed bands to allow wide geographic coverage. Power output ranges from milliwatts typically to about $1 \mathrm{~W}$ for long-range installations. The PAN uses Carrier Sense MultiAccess (CSMA) for network access, and ZigBee extends the PAN by defining a dynamic address scheme that embeds the ad-hoc on-demand distance vector (AODV) mesh algorithm, allowing ad-hoc formation of PANs with dynamic routing. In case of repeater or router failure, the network reconfigures itself after detecting the loss of the existing route. This failover occurs within a few seconds, and provides fault tolerance.

ZigBee was designed around the idea that multiple vendors could interact based on application profiles. Unfortunately much of this interoperability has yet to materialize as vendors provide their own implementations utilizing different power management schemes and message handling on top of the underlying 802.15.4 standard. The ZigBee Alliance is responsible for developing and maintaining the ZigBee protocol and standard.

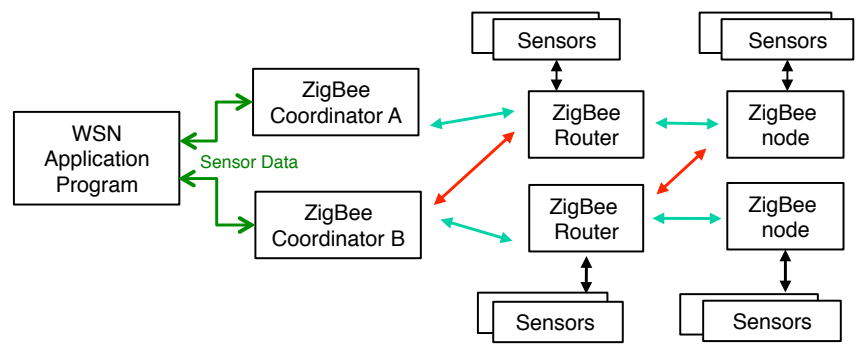

Figure 1. Redundant ZigBee Architecture

The proposed wireless system architecture consists of a network of sensors in a hierarchical and redundant configuration, able to be deterministically configured at run- time and able to respond to sensor module and network faults automatically. This is illustrated in the Figure above. The network would self-configure using the basic mechanisms of the PAN and ZigBee layers. The Endpoints (EP) would be Reduced Function Devices within the ZigBee framework, sleeping most of the time, awakening in time to take the next sensor reading and provide it to the network. The EPs are designed to maximize their battery life by greatly reducing their duty cycle. The main data paths are shown in green, with the failover paths shown in red. The Application Program interacts with the wireless sensor modules using dual-redundant Coordinators. The ZigBee protocol supports automatic retransmission of critical data and automatic re-routing of network paths as well.

\section{SPA OVERVIEW}

The general vision for intelligent spacecraft is that a SPAenabled spacecraft Command and Data Handling (C\&DH) software program can interact with SPA-compatible components forming a complete spacecraft by configuring the data network using the SPA framework at runtime. The spacecraft components are simply provided power, while the SPA-enabled network provides the data paths. The data paths are defined using SPA at system startup using the interactions specified by interconnect-specific subnet manager software. The C\&DH software can then be written to conform to the Spacecraft Data Model (SDM) part of the SPA standard. The ability to re-use large parts of the spacecraft software is one of the chief advantages of this approach, greatly reducing the cost of software development. The vision could go as far as auto-generation of both the spacecraft hardware and software design from a set of high-level spacecraft specifications derived from mission objectives.

There are a number of already existing SPA subnet manager implementations, designated as SPA-S (SpaceWire), SPA-U (USB), SPA-1 (I2C) or SPA-E (Ethernet). SPA already supports the most common networking interfaces for satellite manufacture, which should help it gain traction in the field. The AFRL is working with the AIAA to define formal SPA standards, which have been published. ${ }^{4}$ Example missions such as PnPSat have been implemented with SPA as demonstrations. ${ }^{5}$

SPA incorporates a number of functions to support Plugand-Play. The core components in the SPA network are the Component Addressing Service (CAS), the SPA Lookup Service (LUS), the Local Subnet Protocol Manager (SM-L), and the interconnect-specific Subnet Manager (SM-x). The SPA Processing Node (SPN) hosts various functions: The CAS is the directory of modules and their functions. The SM-L implements the SPA protocol consisting of messages passed between various compliant modules. The SM-X subnet manager handles all the interfaces involved with the logical interconnect. The LUS allows access to xTEDs and sensor data streams. The Figure below diagrams these interactions. 


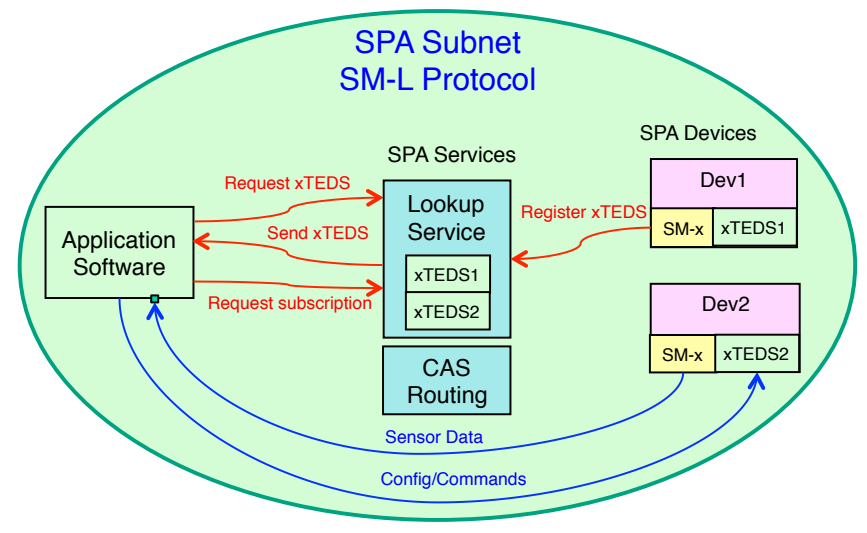

Figure 2. SPA Logical Architecture

Component Addressing Service-The Component Addressing Service or CAS is responsible for assigning unique addresses to all SPA components in the SPA network. In the case of a specific subnetwork such as SpaceWire or ZigBee, this is done through the local Subnet Manager which receives an address block from the CAS. Only one CAS may be operating on the SPA network at a time. The CAS will need to maintain a routing table containing all of the Subnet Managers in the SPA network, storing the object type, its UUID, its assigned address block, and the logical address of each manager. This ensures that all subnetworks with an assigned address block will be routable. It needs to work across hybrid SPA subnets, where one portion uses SpaceWire and the other may use ZigBee.

SPA Lookup Service-The primary purpose of the Lookup Service or LUS is to maintain a directory of all of the components in the system and the services that they provide. It does this by requesting that components register their xTEDS information with the LUS after they have been assigned addresses on the SPA network. The LUS then receives a reply from each component and stores the xTEDS information along with the associated SPA component address so that other applications may query for available services. The LUS must therefore maintain a routing table of services to component addresses in order to respond to such queries.

SPA Subnet Manager-The SM-L implements the messages that implement the SPA protocol. There is a basic requirement that the SPA Subnet Manager be hosted in a computer with significant performance, such as the C\&DH processor, as the protocol requires actions to be taken to complete the configuration and management of the SPA devices in the network.

Subnet Manager-this interconnect-specific manager (SM-x, where $\mathrm{x}$ is the interconnect type) is the program that manages the interaction between the SM-L protocol manager and the subnet itself.

\section{SPA Messaging}

The core concept of SPA revolves around three main ideas:
- Components register themselves with a Lookup Service

- Software applications can perform searches on the registered components in the system

- Components or applications can access the services of other registered components by querying the Lookup Service

Because SPA is primarily a networked data exchange model, the standard also specifies a number of message types that are required for network discovery and transfer of information. The SPA standards are developed to provide a framework by requiring that the request and response message structures needed for this communication is supported by any SPA-compliant components as defined below.

\section{SPA Topology Discovery}

Topology discovery is the method by which components join the network at boot time and retrieve the information necessary to begin the registration process. During topology discovery, the SM-x subnet manager must record the route to all SPA core components, including the CAS, the SM-L and the LUS. It will also discover and record the routes to other Subnet Managers on the SPA network. The following over-the-network data structures must be implemented in order to implement this function.

SPARqstAddrBlock-This message must be sent to the CAS to request an address block from the CAS and assign SPA addresses to the components on its local subnet,. In response the CAS will reply with the SPAAssign-AddrBlock message containing a block of addresses to use.

SPARqstCasRoute-If a component on the subnetwork needs to send a message to a node on another subnet and does not have the route information, the subnet manager uses this message type to request the route from the CAS. The subnet manager must then be able to handle either a SPADistributeRoute or SPAReplyCasRouteUnknown message response from the CAS.

The SPA-Z Subnet Manager must also respond to requests from other Subnet Managers for routing information after topology discovery. In this way all attached subnets will eventually retrieve the routing and addressing information needed to register components.

\section{Component Registration}

In order for components or applications to discover services that are available on the network and connect to them, the available services must first be registered with a central registration authority. Components are required to register the services that they offer with the LUS using Extensible Transducer Electronic Datasheets (xTEDS), an XML version of the TEDS definition found in the IEEE 1451 series of standards. ${ }^{6}$ These services might include the ability 
to publish sensor data at a specified rate or allow the commanding of actuators or setting operating parameters. By publishing these service APIs in XML form to a registration authority, other applications or services can query the registration authority to determine what services and components are available across the network.

The registration process occurs after topology discovery is completed and the core components of the network have been added to the subnet manager routing tables. The local Subnet Manager provides the LUS with the component addresses, after which the LUS will query the component directly. Finally, the SPA device must be able to read the request and respond to the LUS with its xTEDS information. The following message structures must be implementated to complete this process:

- $\quad$ SPAProbeRequest-The LUS sends this request to the component node. The component should reply with a SPA-ProbeReply containing information used to determine network health and status.

- $\quad$ SPAxTEDSRequest-Once the LUS has an entry for the node recorded, it will use the information to send this message back to the component in order to fetch the xTEDS. Finally, the component will respond with a SPAxTEDS-Reply message containing the xTEDS information.

At the completion of this phase, the Lookup Manager should have a record of all components that are currently on-line and also have a copy of the xTEDS for those components.

\section{Subscription Processing}

Once the node services advertised through xTEDS are stored in the LUS, those services may be queried by other components. Services may be queried according to name or qualifiers. In addition, a component may request the metadata for a certain variable or request the entire xTEDS for a component. This functionality requires that another set of request and replies be added to the command set. This is potentially the area of most development for meeting the SPA requirements, since code must be written to parse the individual xTEDS in order to satisfy the search requests.

Because the information requests can also be stored as future subscription requests when other nodes join the network, code must also be written to continually monitor these subscriptions in concert with the state of the network:

- SPARegistrationInfoRequest-This is used to query the list of providers and can be set to receive information when future providers join the SPA network. In response, the SPA LUS will send a SPARegistrationInfoReply message for each match.

- SPAVariableInfoRequest-This command requests additional information about a particular variable stored by the LUS. Similar to the registration info request, the LUS will respond with one or more matches, this time using the SPAVariableInfoReply message type.

- SPASubscriptionRequest-Requests a notification from the service provider. If the provider is able to fulfill the request, it will send a SPASubscriptionReply message to indicate that the subscription was accepted.

After a component has located the information that it is interested in, the LUS may broker a connection between the two components. In this case the SPA LUS will forward the request to the service provider, at which point the two components will begin to negotiate the subscription between themselves. There are a number of options available for the subscription request, including the ability to define lease period, periodic subscriptions, and priority settings.

The remaining message types are primarily for nominal functioning of the network such as data passing and commanding. It is important to reiterate that any commands or data would need to be advertised in the xTEDS for use with any SPACommand or SPAData request:

- SPAData-This message is sent to the component making a data request and the payload contains the data requested as referenced by the xTEDS identification.

- $\quad$ SPACommand-Sent in order to request that another component execute a command, as referenced by the xTEDS identification.

- $\quad$ SPAServiceRequest-The message sent to another component to request execution of a service, as referenced by the xTEDS identification.

\section{SPA-Z DEFINITION}

Wireless sensors have been proposed for spacecraft use with only limited success, as a widely accepted standards-based solution has not been fully developed. While wireless 802.15.4 and ZigBee are not currently being used in spaceflight, we believe that their built-in redundancy and scalability make these standards ideal for certain space applications such as payload subsystems or developmental and flight instrumentation. Integration with a standard such as SPA builds on ZigBee's existing plug-and-play functionality and makes it a logical extension for spacecraft avionics. We are proposing a new standard called SPA-Z for this purpose, and the next several sections will address some of the work that must go into defining this specification for near-term development and eventual adoption as a SPA subnet standard.

The advantages of using the ZigBee standard to implement the key SPA functions of network discovery and management are the ability to build upon the various ZigBee 
protocol implementations provided by a number of commercial vendors. ${ }^{7}$ These ZigBee protocol stacks are complex, but must run on low-performance microcontrollers. ${ }^{8}$ Therefore, the key simplification is to use the underlying ZigBee network management functions for the interconnect-specific subnet manager (SM-Z) while providing the appropriate interface functions to the SPA local protocol manager (SM-L) hosted on the SPA Processing Node.

The SPA-compliant software components would reside on the Full-Function Devices (FFD) such as Routers, Coordinators and Gateways defined within the ZigBee Framework. These FFDs are on continuously and have sufficient computational power for providing key SPA services to other components. It is also likely that the ZigBee Subnet Manager will be the local ZigBee PAN coordinator to facilitate direct interaction between the two functions. The wireless sensor modules (WSMs), acting as EPs, would advertise their services and characteristics via the xTEDs during PAN formation. The SPA services provide the framework for application software interaction with the lower layers. The application software utilizes the SPA framework for interacting with the sensors, sensor modules and network, and can be written in a generic way compliant with the SPA standard. Each WSM, Router, Coordinator and Gateway of the SPA-Z network would have to be SPA-compatible.

The other approach is to adapt a subsystem to SPA using the Applique Sensor Interface Module (ASIM) architecture, where the details of the ZigBee network are obscured by the ZigBee Coordinator/Gateway acting as the interface between SPA and ZigBee. In this case, which best supports highly scalable and low-power ZigBee endpoints, all SPA functions are resident in the Coordinator/Gateway node, which has the processing power to host the SPN and fully implement the appropriate ZigBee to SPA interface translations. In this implementation, the Coordinator/Gateway collects the xTEDS from all ZigBee sensors within the subnet, providing them to the SPA protocol en-masse. It effectively makes a large wireless network look like a single SPA device with hundreds of sensors. The approach has the advantage of scalability to a large number of ZigBee nodes and also minimizes the software changes to the ZigBee endpoints.

The proposed SPA-Z implementation of these core components requires that a ZigBee Subnet Manager be instantiated as a full-function device. This should be the only piece of new software requiring development. The other SPA services would have to be hosted on suitable processors and the appropriate source for the software found. It is likely that the Subnet Manager will be hosted on the local ZigBee PAN coordinator. One can either implement the SPA-Z Subnet Manager on all nodes of the wireless network or more simply on a single node.

In our SPA-Z prototype implementation we will use the Network Capable Application Processor (NCAP) device, (an IEEE 1451 legacy) which also serves as the PAN coordinator for the ZigBee network, to host most of the new SPA functionality rather than distributing these functions across the ZigBee network. This approach can considerably simplify the amount of new software that needs to be written and conforms to the ASIM architectural approach. Note that once a suitable SPA-Z Subnet Manager has been implemented, it could be duplicated on all the ZigBee nodes, resulting in a fully SPA-compliant architecture. Therefore, the ASIM approach allows early prototypes to be extensible to the more capable architectures.

\section{ZigBee to CAS Addressing}

One of the first interface problems between ZigBee and SPA that needs to be addressed is the translation between ZigBee Addresses and CAS Addresses. There are two specific approaches for solving this problem: the first would be to translate the ZigBee node addresses directly to CAS addresses. However, since the ZigBee addressing scheme is not constant (node addresses can change during network reconfiguration) this approach has significant drawbacks. Our team actually embedded absolute addresses into the ZigBee modules to solve this problem in the past, and a similar approach would work well. A block of SPAcompliant addresses would be written into the non-volatile memory of the ZigBee devices and used for SPA CAS registration. These addresses would be accessed using the appropriate SPA addressing messages.

Note that while ZigBee addresses are actually used by the ZigBee protocol for routing, the CAS addresses are the actual module addresses used for SPA functions. The CAS addresses become Application Support Layer attributes of the ZigBee Framework, and therefore remain consistent. Because the CAS addresses are represented as higher-level ZigBee node identifiers, they remain the same despite wireless network reconfiguration occuring at the PAN and ZigBee levels.

\section{Generating $x T E D S$}

The SPA standard specifies that extensible Transducer Electronic Datasheets (xTEDS) be used to register application services at the end-nodes. The xTEDS contains the key meta-information required for both SPA network management and for application interaction with the SPA device's functions such as sensor data output or control actuator inputs.

The xTEDS is an extension of the TEDS defined in the IEEE 1451.0 standard and relates to earlier project work. We had generated TEDS datasheets as part of our project last year, so converting those to XML format for XTEDS was relatively straightforward. The capability of embedding control commands and network management features into the XTEDS is a major improvement over TEDS. However, the format for xTEDS is much less restrictive and subsequently poses some additional challenges. While the xTEDS XML schema defines the attributes and elements that are allowed in the XTEDS, it does not specify the set of 
values to be used for those attributes or elements. An example of the problem this might cause can be seen in the type field - one vendor might generate an xTEDS datasheet that specifies the measurement type as "temperature", while a second vendor uses the keyword "Temp" for a similar sensor. If a third-party application were to query the SPA LUS for temperature sensors it might get an incomplete set of results. A related problem might arise from the attribute type for this sensor. If engineering units are returned, one vendor may record the temperature in degrees Fahrenheit while another uses Celsius.

The solution to this problem is the maintenance of a Common Data Dictionary (CDD) for storing and managing these terms. The Consultative Committee for Space Data Standards (CCSDS) recommends the use of an information architecture to manage these terms locally and to allow for domain-specific meta-models to be created and shared across working groups. This would allow disparate groups within the same domain to better exchange and compare datasets, and also presumably to build up a suite of tools to better examine these datasets. To this end, the CCSDS has created an abstract reference architecture for creating and defining information objects in the context of a larger domain model. ${ }^{9}$ Some examples of domain models that have already been created include the Earth Observing System Data and Information System (EOSDIS) and the Space Physics Archive Search and Exchange (SPASE).

For our project, we use the CCSDS Spacecraft Onboard Information Systems (SOIS) Device Virtualization Service (DVS) standard in the creation of a common dictionary. The CCSDS SOIS is recommended for internal spacecraft communications by the SPA Standards Development Guidebook, simplifying our decision to use this related standard. In reality, the SOIS standard provides a full array of specifications for ZigBee's Application Support Layer Services. We build on top of the existing SOIS DVS definition in order to add the ZigBee-specific management tools. For the purposes of our testbed an xTEDS file was generated for a simple thermistor. For more on the generated xTEDS and constructing a CDD of terms for the xTEDS, see Appendix A.

One thing to note in the example above is that one xTEDS may represent multiple sensors located on a single endpoint. The ability to add many different services to one node is especially suited to ZigBee using the concept of application profiles. ZigBee allows up to 240 application profiles to exist on a single wireless node. Creating a ZigBee device profile by adding one or more ZigBee cluster libraries around the concept of spacecraft management could potentially enable these components to connect and communicate with each other with very little outside integration. According to the ZigBee Cluster Library specification, a ZigBee cluster is defined as a related collection of attributes and commands, which together define a communications interface between two devices.
The devices implement server and client sides of the interface respectively. Examples of cluster commands might include operating actuators, firing thrusters or publishing sensor values across the system. Further work in using these high-level ZigBee functions to simplify SPA adaption will be initiated in the future.

\section{Data distribution system (DDS)}

The LUS software responsible for xTEDS registration and Subscription Processing could be combined with a Data Distribution System (DDS) in order to take advantage of the benefits that DDS has to offer. DDS is a publish/subscribe architecture that has been specified by the Object Management Group specifically for data dissemination in real-time and embedded systems. ${ }^{10}$ The publishers and subscribers in the network share information by using an application-defined name or Topic and a key associated with that Topic. Because these shared names are application-defined, they could easily be specified in the xTEDS associated with a given sensor. The subscription process would then be more robust and with several built-in controls. Among these, the ability to add Quality of Service (QoS) parameters to improve network response time or provide content-based subscriptions is extremely useful and potentially necessary for any level of hardware-in-the-loop flight-control. Other benefits that DDS offers include builtin control over data persistence and data queuing, which is of particular importance in mesh networking where a node could drop out of communication and rejoin some time later. Finally, the use of DDS may actually reduce the lines of code needed for a working SPA implementation since much of the functionality for subscription processing would be implemented in the DDS software. Others have used DDS as the foundation for SPA implementations.

\section{ZigBee Service Interface}

In addition to the interface specification added for each sensor on a ZigBee node, there is information associated with the wireless interface itself that must be managed. This proposed ZigBee service interface was derived from the Spacecraft On-Board Interface Services-Device Virtualization Service Draft Recommended Standard CCSDS 871.2. The ZigBee service interface provides ZigBee class-specific service primitives to access functions common to all ZigBee wireless nodes. The DVS_GET_ZB_STATUS.request message is used to request the status of a ZigBee node and returns parameters such as: Device_ID identifies the ZigBee node; RSSI is the current link quality measurement; Battery_Status is the current state of the ZigBee battery, in estimated percentage remaining; Architecture is the current ZigBee network hardware architecture and TIM Type is the type of ZigBee node with possible values being coordinator, router or enddevice.

\section{SPA-Z FOR SPACECRAFT}

A simple example of a SPA-based spacecraft will illustrate the utility of wireless networks combined in a hybrid 
configuration with SpaceWire. Consider the need for providing flexible payload accomodations provided by wireless SPA-enabled subsystems. Figure 3 depicts a simple spacecraft bus with its major components connected by SpaceWire. The SpaceWire segment connects the Command and Data Handling (C\&DH) control computer to the Electrical Power Subsystem (EPS) and Attitude Control Subsystem (ACS), which together provide basic flight functions. This segment could be SPA-compliant or not, as we are only interested in the payload functions at this time. The adoption of SPA for various Aerospace functions such as CubeSats is evident by product support from Companies such as AAC Microtec. ${ }^{11}$

This example illustrates a spacecraft where all Payload functions are wireless SPA compliant, with only physical mounting and power connection required for spacecraft payload configuration. Examples of missions where this might be favorable would be constellations of earth observation satellites (A-train) or multi-point physics science missions. We consider the Payload to be an instrument together with an optional GPS unit and the appropriate Communications Processor for downlink. The spacecraft may have separate command and telemetry (C\&T) links, but for this example we use a dedicated downlink for payload data to better demonstrate modular Plug and Play technology. The objective is to support multiple modular functions that complement each other and have to interact intimately using SPA as the physical and logical interconnection.

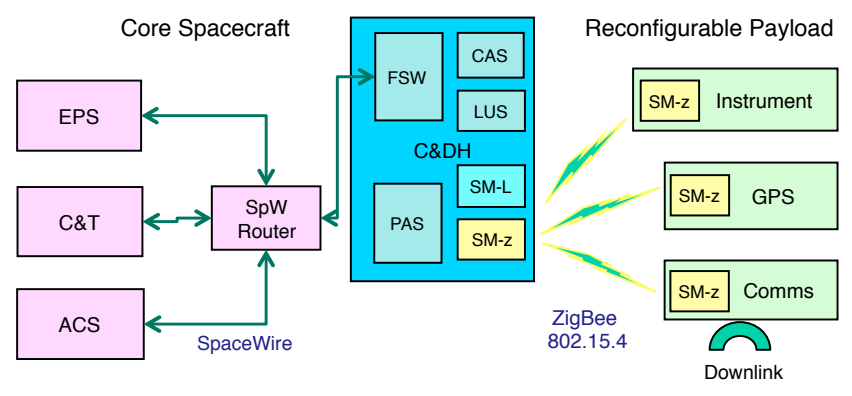

\section{Figure 3. Wireless SPA Spacecraft}

In this example, the core spacecraft functions (C\&DH, EPS, C\&T and ACS) connect using SpaceWire. The C\&DH runs the Flight Software (FSW) and can provide an interface to SPA. The SPA Services are hosted in the C\&DH computer, and provide the interface to the SPA-compliant components. Therefore, the C\&DH has to host the LUS, CAS and SM-L protocol functions. The C\&DH also has to host the SM-Z subnet manager together with the ZigBee physical interface. However, these are the only spacecraft modifications needed for supporting a SPA-enabled payload.

The payload Instrument, GPS and Comms Processor (Comms) components are all SPA-compliant devices which interact with the C\&DH hosted SPA services using the standard SPA messages over the ZigBee link. Each component has a rather large xTEDS describing the software interface functions for the entire subsystem. Each SPA Device (in this case a spacecraft subsystem) would register its xTEDS with the LUS, setting the stage for payload operation by the Payload Application Software (PAS). The PAS is the only custom component needed to implement a custom payload function and is hosted within the $\mathrm{C} \& \mathrm{DH}$ as an application-layer program. The PAS is SPA-aware and must use the SPA protocols to interact with the payload components.

The PAS polls the LUS until the SPA-components are registered, then parses the XTEDS to identify the specific devices to be used and the software interface (cmds, sensor data) to operate the component. For example, the PAS would recognize the GPS and subscribe to its coordinate updates. The PAS would use the coordinates to initiate a downlink to a specific ground station using the Comms Processor xTEDS to understand the methods available. The PAS would obtain the data for downlink from the payload Instrument using the xTEDS describing the instrument's command set and status indications. The embedded software interface represented by the meta-information in the xTEDS is the core of SPA function, providing a method for implementing a general software API for reconfigurable payloads.

Therefore, a spacecraft could be rapidly configured for a given mission by physically loading the Payload Devices, connecting power and running the PAS program in the C\&DH. The SPA Services provide the interaction between the core spacecraft and the PAS program but do not have to be changed to reconfigure the spacecraft. It is anticipated that a "generic" PAS, supporting multiple configurations, could be written, thereby eliminating the need to change even the Payload software, leading to effective software reuse and the resultant cost savings. This is of particular benefit when constructing multiple similar spacecraft requiring different payloads.

In this example, the ZigBee subnet does not have many nodes, may or may not support redundancy and is therefore considered a simple wireless network configuration. The major benefit of using wireless for this purpose is to eliminate the data cables required to run the payload and downlink components, a very modest gain. However, for CubeSats, this cabling may be a rather large percentage of available spacecraft resources. Therefore, the real benefit may be supporting more complex functions on smaller spacecraft.

To implement such a flexible payload system, the reconfigurable payload components would all have to be SPA-compliant. This would require the development of a SM-Z subnet manager for ZigBee. It would require implementing the SPA protocol (SM-L) in the ZigBee interface software, enabling the xTEDS registration process and middleware-mediated data exchange. The best approach for this example would be to implement the SPA protocol 
using ZigBee DVS messages in the wireless module's firmware. Note that each ZigBee module has to host the SPA-Z Subnet Manager, the SPA Local Protocol (SM-L) and the xTEDS specific to its function.

\section{DeVELOPMENTAL AND FLight INSTRUMENTATION SYSTEM}

Developmental and Flight Instrumentation (DFI) using wireless sensor networks (WSN) can lead to a signficantly lower cost per sensor than conventional wired approaches while providing the benefits of reduced cable mass and flexibility of placement, both important attributes for flight vehicle instrumentation. Less obvious are the developmental and operational cost savings possible through the use of Plug-and-Play standards-based technology, which enables multiple vendors to produce compatible products and even generic application software able to support a wide-range of configurations addressing a broad range of aerospace needs. Lastly, since wireless data communication is immune to cabling and connector faults, it also provides a diverse method for DFI system fault tolerance.

Earlier prototype work by the team for DFI used ZigBee wireless components within an architecture compliant with IEEE 1451, which defines the Network Capable Application Processor (NCAP) as the primary computing platform with the Telemetry Interface Modules (TIM) hosting the sensors. During earlier evaluation of standards and prototype ZigBee products for DFI, certain deficiencies were noted in current technology and standards. ZigBee node addressing is route dependent and can change during network reconfiguration or initialization. Therefore, a unique identifier is needed to support deterministic sensor networks to resolve this ambiguity. Embedding SPA addresses in each ZigBee module can address this need. The TEDS templates needed far more flexibility, hence the move to xTEDS. Time stamps can be incorporated in the network data flow using ZigBee Global time as its reference source. Finally, no module or component should limit overall throughput, so routers and gateways need to be designed to support the full data rate.

There is no clear consensus on application software access to streaming data values and network parameters for WSNs. Current implementations use SNMP servers or Pythonbased APIs for application software interfaces, and each method suffers from throughput limitations or implement a proprietary API. ${ }^{12}$ The IEEE 1451 has been used for defining sensor parameters using its Transducer Electronic Data Sheets (TEDS) which were the precursor to the XMLbased TEDS (xTEDS) used by SPA. As described below, the xTEDS for a given sensor will specify the type of data that it can publish or receive via the SPA LUS. The project also determined that the use of standard publish/subscribe middleware techniques might be the best fit for the application software interface implementation, and identified DDS as the best approach. The team recognized that its approach to improving WSN system design was very similar to the methods identified by SPA, resulting in this paper, which is both a guide for developing wireless SPA standards as well as SPA-compliant WSN implementations.

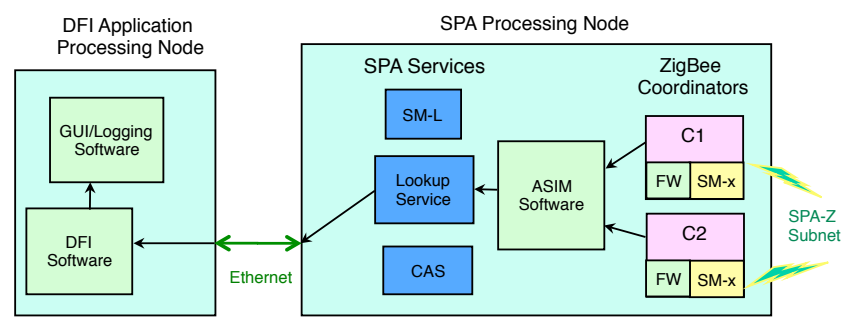

\section{Figure 4. DFI ASIM Block Diagram}

\section{Proposed DFI architecture}

We propose modifying the basic IEEE 1451 architecture by using the NCAP processor to create the SPA Processing Node (SPN) because the NCAP contains sufficient processing power and is the primary bridge between application software and the wireless sensor modules (WSM), acting as TIMs. The proposed architecture for our DFI prototype implementation is to use the NCAP gateway as the host for SPA functions using the ASIM approach. Beyond the NCAP, the rest of the WSN functions can use the native ZigBee and 802.15.4 protocols. The major reason for this approach is to utilize the built-in network discovery, addressing and routing functions of the ZigBee protocol, which were developed specificially for wireless and which function very well. Note that one cannot use both SPAbased network discovery and management methods simultaneously with ZigBee, as the two functions will conflict. One must choose one approach or another and the use of the ZigBee network formation and management processes is the correct approach in this case.

This architecture does not require complete modification of the ZigBee endpoint or router firmware, its chief advantage. All SPA functions are embedded in the ASIM adapter hosted in the SPN, which functions as the root of the sensor network and the gateway to the rest of the spacecraft systems. The key endpoint modification is to insure that each WSM contains the appropriate XTEDS describing its functions along with the methods for reading the xTEDs upon initialization or reconfiguration, functions provided by SPA-Z. The SPN aggregates all xTEDs into the resident LUS and presents the complete set to the application software through the DDS-based LUS. Therefore, the entire WSN becomes a single SPA device hosting many xTEDs respresenting the entire WSN sensor complement.

Starting from an interesting combination of ZigBee and IEEE 1451, the team mapped these functions to SPA, resulting in a roadmap for further definition. The next steps were definition of sensor and network descriptions in xTEDS, the modification of existing software to support variable-field XML descriptions, and the definition of SPA messages to help control the network and sensor modules. The source and version of DDS software and other SPA 
support services were then identified to help define the implementation plan.

\section{Network Discovery Implementation}

The use of the underlying ZigBee network management function means that the only new software needed is the interface between the SPA discovery functions and the embedded ZigBee network functions. Many of these functions are simply translations of ZigBee Application Support Layer identifiers and status messages to the SPA standard, which should be rather simple.

The ZigBee coordinator(s) resides in the SPN, which hosts the SPA services and also performs the data gateway functions. At run-time, the coordinator will create the PAN, dynamically assigning ZigBee addresses to each WSM in the wireless network. It also creates all the routing tables needed to initiate data transfer. The coordinator then passes this network configuration information to the host processor for translation to SPA CAS addresses and network status messages. Dynamic reconfiguration requires that these processes remain active to support any changes to WSM status or network configuration occuring after initiation.

\section{xTEDS Aggregation}

Each WSM contains a specific xTEDS describing its sensor complement. The SPN should aggregate all xTEDS for every WSM in the DFI network. It does this by transfering the xTEDS from the WSM to the SPN using the underlying ZigBee data transfer functions called from SPA-Z. The SPN therefore ends up with the full set of xTEDS representing every sensor in the network. Note that xTEDS registration and de-registration is dynamic and driven by SPA network discovery and management functions. A method of connecting these SPA functions to the ZigBee network functions would have to be implemented in SPA-Z.

\section{Middleware Implementation}

The Data Distribution Service (DDS) as specified by the OMG is the chosen publish/subscribe standard. DDS uses topics to partition the data being sent across the middleware, so that an end user might specify a group of nodes by selecting that specific topic to subscribe to. In effect, DDS middleware abstracts the source and details of data and status delivery, making them software objects conforming to certain defined categories, and providing topic query functions. Therefore, the use of DDS provides many required SPA LUS functions, requiring just a modest amount of new software to reconcile the functions.

The mechanism for delivering ZigBee sensor data to a requestor could easily be satisfied through the use of topics using the xTEDS-defined sensor type as a topic name. The commands defined for a specific ZigBee node/interface may also be sent through DDS in order to change the data rates or to command actuators. Finally, the network management commands needed for SPA configuration could also be encapsulated in a DDS topic. Therefore, the DDS-based LUS would provide xTEDS access, create sensor data topics containing the real-time sensor data and create network and module status topics for WSN management.

One example of an attempt to create a more general approach outside of the ZigBee framework is TinyDDS, which provides protocol and programming language interoperability across the ZigBee gateway. ${ }^{13}$ TinyDDS is one of the few implementations that is designed for lowpower, low-memory devices and can sit on top of the ZigBee stack, but is only available for Mica-Z and SunSPOT platforms as of the time of this paper. Still, the use of DDS was identified as the best approach for this middleware function because of its near real time delivery and cross-compatibility between vendors. We also experimented with OpenSplice DDS Community Edition, and RTI Connext DDS, both open-source communitysupported implementations.

\section{Redundancy and Fault Tolerance.}

The example DFI system provides fault tolerance through redundancy of sensors, modules and wireless network interconnects. The ZigBee protocol provides dynamic network management, reconfiguration and retransmission. Using this approach, the SPN would contain at least two ZigBee coordinators to provide single-fault tolerance. Managing the redundant coordinators has to be done in the host processor, which could use various schemes common to aerospace. For example, one coordinator could be used as a hot backup, enabled after detection of primary coordinator failure. A more complex scheme would be the use of both coordinators to load-balance the network by running in parallel. In the event of failure, the remaining coordinator would have to handle the full network load.

This redundancy would be managed by the SPN and the details obscured to the SPA interface, perhaps just providing an additional status parameter to the Application Layer indicating when redundancy has been lost.

\section{Conclusions}

Our work is a preliminary trade study that can be validated upon implementation and evaluation of a reference design. The objective is to define an extension to SPA Standards for wireless ZigBee networks by defining the functions of the SPA-Z Subnet Manager software. Therefore ZigBee would be another SPA supported interconnect able to interact with other SPA compliant components. This allows the full range of benefits provided by wireless networks to be realized within the SPA ecosystem.

Several standards are involved in the technical approach to this problem. In additional to SPA and ZigBee, the IEEE 802.14.5 PAN standard and IEEE 1451 Smart Transducer standards are relevant and foundational. Further work by CCSDS on the SOIS DVS standards can improve overall applicability by creating a common ontology for device 
identity and description. The IEEE 1451 standard provides good background for construction of xTEDS descriptions from defined TEDS templates taking advantage of IEEE 1451 command sets and architectures. Finally, emerging SOIS DVS standards help define effective methods for managing wireless networks and modules, as well as work defining a Common Data Dictionary for specific application domains.

The approach is to use the appropriate elements of each standard to create a reference design. This is similar to a rapid prototyping approach, which often yields the best implementation at the lowest cost, but at the expense of fully complying with the selected standards. We did not identify any basic problems integrating the various elements of the chosen standards, but also saw that only certain portions of the standards were truly relevant to the integrated product. While definition of standards is often approached from the academic perspective of identifying requirements and functions, the experimental approach of creating a reference design using available building blocks often produces the most optimal implementation in the shortest time frame.

Prior work identified shortfalls of current WSN technology that can be addressed by using SPA features to significantly increase utility: The use of xTEDS allows flexible sensor and actuator definitions and the incorporation of multiple network and module parameters for WSN management; The use of DDS middleware to publish real-time data streams to multiple applications provides a flexible standard for modern software architecture; The use of SPA messaging allows interoperability with other SPA components, increasing the breadth of applicability and enabling interoperatiblity between vendor products.

Two basic approaches to SPA-Z architecture were identified: the use of SPA messages embedded in the ZigBee protocol layer and implemented in the module firmware; or the use of the ASIM Adapter approach. For spacecraft subsystems, it may be better to fully embed SPA within the entire wireless networks. This allows each wireless module to stand-alone within a SPA system. For DFI applications, consisting of many simple wireless components, the use of ZigBee routing ensures scalability and moves the complicated functions to the ASIM Adapter, greatly reducing resource demands on each WSM. This reduces the resources needed at the sensor modules, lowering size, power and cost. However, only the ASIM Adapter hosted in the SPN can interact with other SPA components. This architecture is similar to the NCAP and conforms to IEEE 1451.
The main issue for SPA-Z consists of understanding the best approach for using the underlying PAN and ZigBee protocol functions effectively to implement the reference design. It appears that using the ZigBee Application Support Layer to implement the CAS addressing and SPA messaging functions may be the most effective integration path. This and the addition of the xTEDS to each module implements the key SPA functionality needed in each wireless module. The other issue is hosting the SPA services within the overall system architecture.

It is desirable to create a standard implementation for the entire ecosystem of devices that are used on a spacecraft or satellite. The CCSDS SOIS standards could help establish this by creating common definitions and messages. There are several common components that are expected to be present for navigation, orientation and power management tasks. Creating true Plug-and-Play interfaces for these devices where common requests and attributes are specified in the architecture would further simplify integration between multiple vendors and enhance testability.

It is very important to reduce the cost and complexity of Aerospace vehicle integration, as this tends to be a major cost driver. The capability of using standard software interfaces allows much of the application software to be reused for multiple purposes, reducing cost, but also making a larger range of application software available for spacecraft. The trend for modern hardware and software is toward ad-hoc dynamically configured network systems, which by their nature, change the approach used for application software implementation. The team has found that such dynamic systems must incorporate the mechanisms supporting reconfiguration at every level of the system from the physical interconnect to the application sofware. SPA standards lead the way to achieving such adaptable intelligent systems and integration with wireless ZigBee networks appears to be possible and desirable.

\section{ACKNOWLeDGeMents}

The authors wish to thank Daniel Winterhalter, Nans Kunz, Fernando Figueroa and Ann Patterson-Hine of NASA for their support of this work. Also technical contributions from Mobitrum Corporation working under a NASA SBIR grant were used for the initial WSN prototype evaluation work. Finally, Pete Wilson provided much support for ZigBee hardware and software implementation. 


\section{Appendix A: xTEDS example Thermistor}

<xTEDS xmlns=http://www.interfacecontrol.com/SPA/xTEDS xmlns:xsi="http://www.we.org/2001/XMLSchema-instance" xsi:schemaLocation="../Schema/xTEDS02.xsd" name="thermistor.xTEDS.xml" version=" $2.5 ">$

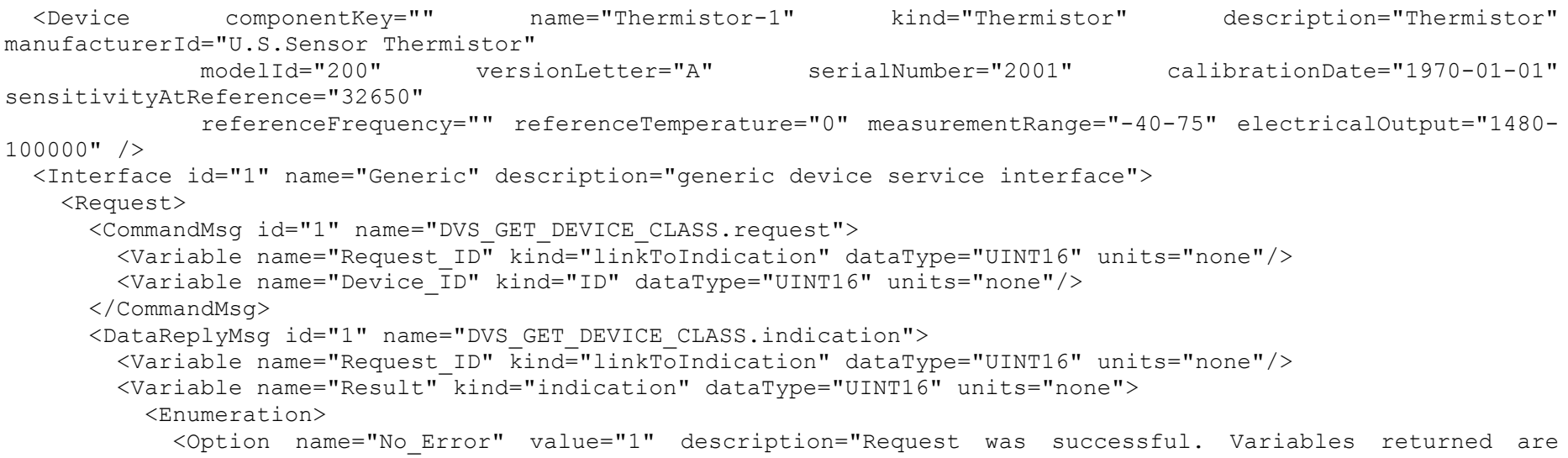


passed." />

<option name="Initializing" value="2" description="Initialization is in progress."/>

<option name="Initialized" value="3" description="Device is in operational or standby mode."/>

coption name="Failed" value="4" description="An internal check during power switch on has not

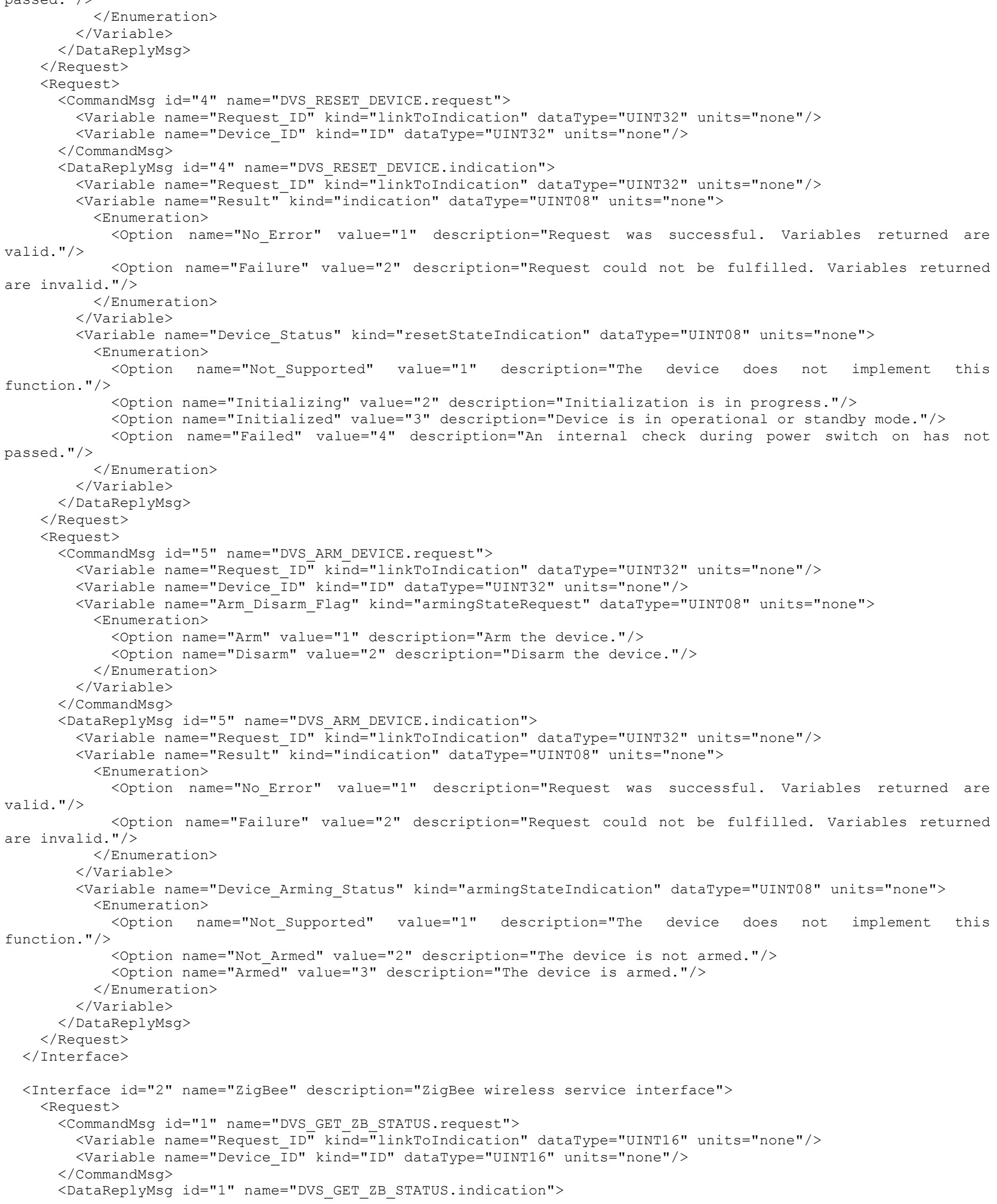


<Variable name="Request ID" kind="linkToIndication" dataType="UINT16" units="none"/>

<Variable name="Result" kind="indication" dataType="UINT16" units="none">

$<$ Enumeration>

<option name="No_Error" value="1" description="Request was successful. Variables returned are

valid."/>

<option name="Failure" value="2" description="Request could not be fulfilled. Variables returned are invalid."/>

$</$ Enumeration $>$

$</$ Variable $>$

<Variable name="Device ID" kind="ID" dataType="UINT16" units="none"/>

<Variable name="RSTSI" kind="signalStrength" dataType="UINT16" units="none"/>

<Variable name="Battery_Status" kind="batteryLife" dataType="UINT16" units="none"/>

<Variable name="Architecture" kind="hardwareArchitecture" dataType="UINT08" units="none"> $<$ Enumeration>

<Option name="cc2430" value="1" description="The device is a TI cc2430 board"/>

<option name="cc2530" value="2" description="The device is a TI cc2530 board"/>

$</$ Enumeration $>$

$</$ Variable $>$

$<$ Variable name="TIM Type" kind="nodeType" dataType="UINT08" units="none">

$<$ Enumeration>

<option name="coordinator" value="1" description="The node is acting as a ZigBee coordinator."/>

<option name="router" value="2" description="The node is acting as a ZigBee router."/>

device."/> <option name="end-device" value="3" description="The node is acting as a zigBee end-

$</$ Enumeration $>$

$</$ Variable $>$

$</$ DataReplyMsg $>$

$</$ Request $>$

$</$ Interface $>$

<Interface id="3" name="Thermistor" description="Thermistor service interface">

$<$ Request $>$

<CommandMsg id="1" name="DVS_GET_TEMPERATURE.request">

<Variable name="Request_ID" kind="linkToIndication" dataType="UINT32" units="none"/>

<Variable name="Device_ID" kind="ID" dataType="UINT32" units="none"/>

$</$ CommandMsg $>$

<DataReplyMsg id="1" name="DVS GET TEMPERATURE.indication">

<Variable name="Request_ID" kind="linkToIndication" dataType="UINT32" units="none"/>

$<$ Variable name="Result" kind="indication" dataType="UINT08" units="none">

$<$ Enumeration>

valid."/>

<option name="No Error" value="1" description="Request was successful. Variables returned are

are invalid."/>

<option name="Failure" value="2" description="Request could not be fulfilled. Variables returned $</$ Enumeration $>$

$</$ Variable $>$

<Variable name="Temperature" kind="temperature" dataType="FLOAT64" units="F" rangeMin="0"

rangeMax="100" accuracy="0.10" />

<Variable name="Thermistor_Status" kind="thermistorStateIndication" dataType="UINT08" units="none"> $<$ Enumeration>

<Option name="No_Error" value="1" description="The device has detected no errors."/>

<option name="Error" value="2" description="The device has detected an error."/>

$</$ Enumeration $>$

$</$ Variable $>$

$</$ DataReplyMsg $>$

$</$ Request $>$

$<$ Request $>$

<CommandMsg id="2" name="DVS_GET_SENSOR_RATE.request">

<Variable name="Request ID" kiñd="lin k̂ToIndication" dataType="UINT32" units="none"/>

<Variable name="Device_ID" kind="ID" dataType="UINT32" units="none"/>

$</$ CommandMsg $>$

$<$ DataReplyMsg id="2" name="DVS_GET_SENSOR_RATE.indication">

<Variable name="Request ID" kind="linkTöIndication" dataType="UINT32" units="none"/>

<Variable name="Result" kind="indication" dataType="UINT08" units="none"> $<$ Enumeration $>$

<option name="No_Error" value="1" description="Request was successful. Variables returned are

valid."/>

<option name="Failure" value="2" description="Request could not be fulfilled. Variables returned are invalid."/>

$</$ Enumeration $>$

$</$ Variable $>$

<Variable name="Sensor Publish Rate" kind="duration" dataType="FLOAT64" units="s"/>

$</$ DataReplyMsg $>$

$</$ Request $>$

$<$ Request $>$ 


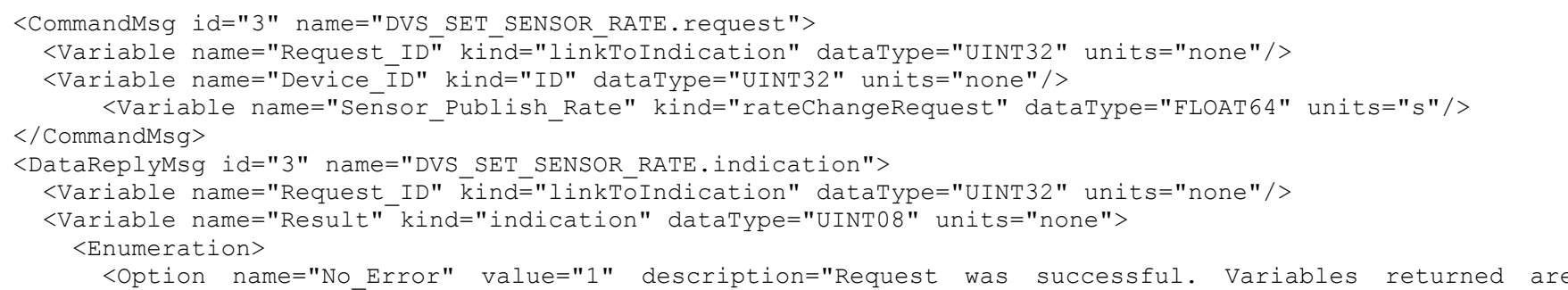




\section{REFERENCES}

[1] Alena, R., Gilstrap, R., Baldwin, J., Stone, T., Wilson, P.,"Fault Tolerance in ZigBee Wireless Sensor Networks,” IEEE Aerospace, 2010 P1480-5

[2] Alena, R. Figueroa F., Ossenfort J., "Intelligent Wireless Sensor Networks for Spacecraft Health Monitoring," AIAA Infotech Conference 2012

[3] Complete standard documents and white papers are available at the ZigBee.org web page: http://ZigBee.org/Standards/ZigBeeNetworkDevices/Over view.aspx

[4] AIAA G-133-1-2013, Space Plug-and-Play Architecture Standards Development Guidebook, Published 2013

[5] Plug-and-Play (PnP) Structures for Satellite Applications, http:/www.afsbirsttr.com/Publications/Documents/Innov ation-042309-SpaceWorks-AF06-273.pdf

[6] IEEE 1451 URL:

http://www.nist.gov/el/isd/ieee/ieee1451.cfm

[7] Z-stack reference URL: http://vast.uccs.edu/projects/traumagps_files/docs/chipcon /Z-Stack.pdf

[8] CC2530 Reference URL: www.ti.com/lit/ds/symlink/cc2430.pdf

[9] Reference Architecture for Space Information Management, CCSDS 312.0-G-0 Green Book, March 2013.

[10] DDS reference URL: portals.omg.org/dds/

[11] AAC Microtec reference URL: http://pnp.aacmicrotec.com/index.php/introduction-toplug-and-play.html

[12] Digi reference URL: http://www.digi.com/products/ZigBee/

[13] P. Boonma and J. Suzuki, "Toward Interoperable Publish/Subscribe Communication between Wireless Sensor Networks and Access Networks," In Proc. of IEEE International Workshop on Information Retrieval in Sensor Networks (IRSN), Las Vegas, NV, January 2009.

\section{BIOGRAPHY}

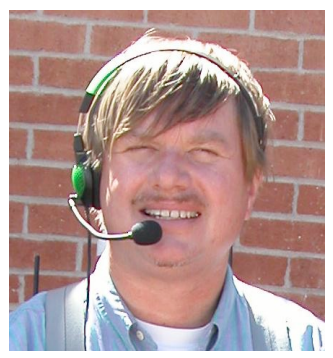

Richard L. Alena is a Computer Engineer in the Intelligent Systems Division at NASA Ames. Mr. Alena worked on the Ground Data System and performed Communications Analysis during operations for the LCROSS Lunar
Mission and on avionics and software architectures for Lunar Surface Systems for human missions. He was the colead for the Advanced Diagnostic Systems for International Space Station (ISS) Project, developing model-based diagnostic tools for space operations. He was the chief architect of a flight experiment conducted aboard Shuttle and Mir using laptop computers, personal digital assistants and servers in a wireless network for the ISS. He was also the technical lead for the Databus Analysis Tool for International Space Station on-orbit diagnosis. He was group lead for Intelligent Mobile Technologies, developing planetary exploration systems for field simulations. $\mathrm{Mr}$. Alena holds an M.S. in Electrical Engineering and Computer Science from the University of California, Berkeley. He is the winner of the NASA Silver Snoopy Award in 2002, a NASA Group Achievement Award in 1998 for his work on the ISS Phase 1 Program Team and a Space Flight Awareness Award in 1997.

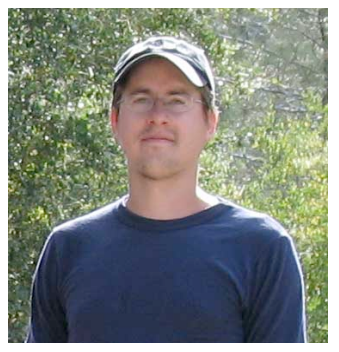

John Ossenfort is a Computer Scientist and employee of SGT, Inc. at NASA Ames Research Center. He is currently working in the Discovery and Systems Health research area, integrating fault management technologies with advanced testing and demonstration of the Orion Multi-purpose Crew Vehicle. In the past he has worked in networking and systems administration on several exploration projects and participated in various field simulations, assisting in all aspects of wired and wireless network design, deployment, troubleshooting and maintenance. John has a dual BA degree in Anthropology and East Asian Studies from Washington University in St. Louis.

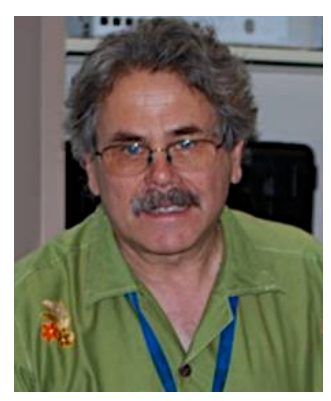

Thom Stone is a Senior Computer Scientist with Computer Sciences Corp. He got his Bachelors degree at SUNY Stony Brook. Mr. Stone has been at NASA ARC employed by various contractors since 1989 working on advanced networking. He was an engineer with the NASA Science Internet project office where he led the project that brought reliable Internet connections to remote locations including U.S. bases in Antarctica including McMurdo Station and Amundson Scott South Pole Station. He was principal engineer for communications for the NASA Search for Extraterrestrial Intelligence (SETI) project and was a senior engineer for the Space Station Biological Research Project. Before his involvement with NASA, Stone was employed in the computer and communications industry and taught telecommunications at the undergraduate level. 


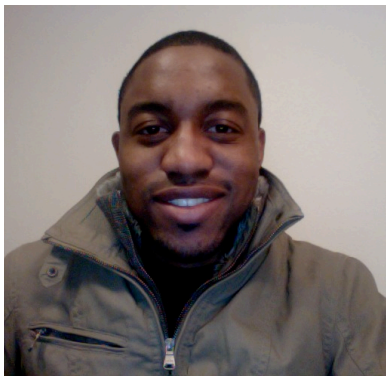

Jarren A. Baldwin is a Chicago native and currently serves as the lead Electrical Engineer at Bay Area startup, Oculeve Inc. He graduated from the University of Illinois with a B.S. in 2009 and received an M.S. in Electrical Engineering from Stanford University in 2012.

Jarren developed hardware and software systems for a wide range of fields, including space science systems and medical devices as a NASA Ames intern in the Intelligent Systems Division. 